\title{
Fetal Doppler Simulator Based on Arduino
}

\author{
Alfina Nadhirotussolikah", Andjar Pudji, M. Ridha Mak'ruf \\ Department of Electromedical Engineering Poltekkes Kemenkes, Surabaya \\ Jl. Pucang Jajar Timur No. 10, Surabaya, 60245, Indonesia \\ \#alfinanadhirotussh55@gmail.com, andjar.pudji@gmail.com,m.reedha@gmail.com, \\ Article info: Received May 24, 2019; Revised July 4, 2019; Accepted January 6, 2020
}

\begin{abstract}
Heart rate of fetal is the main indicator of fetal life in the womb. Monitoring fetal heart rate can't be done, so a tool is needed to monitoring fetal heart rate. Fetal heart rate can be monitored with fetal doppler. To test the accuracy of Fetal Doppler, a calibration is needed with the Fetal Doppler Simulator. This tool will simulate the fetal heart rate with a BPM value that can be adjusted according to the settings on the device. This module using Arduino as the brain system. On the module, there is a selection of BPM from 60 to 240 BPM with an increase of 30 BPM displayed on 2x16 character LCDs. Based on BPM measurement 6 times using Fetal Doppler, the measurement error in a BPM of 60 to BPM 210 is $0 \%$, while at BPM 240 an error is $0.2 \%$. This module has been compared with the standard devices (Fetal Simulator Brand Fluke Biomedical Ps320), the results of the comparison modules with the comparison tool has the same error value in 240 BPM is $0.2 \%$ and in BPM 210 there is a difference in the result of module Fetal Doppler reading of 210 BPM while in the comparison tool is 209 BPM. Of the measurement data and analysis, it can be concluded that the tool can work and the tool has the same accuracy as the standard device.
\end{abstract}

Keywords-Fetal Heart Rate; BPM; Fetal Doppler Simulator

\section{INTRODUCTION}

Fetal heart rate (FHR) is a sensitive indication of fetal status, especially because it is associated with uterine contractions [1]. Monitoring fetal heart rate cannot be done in plain view, so equipment is needed to help monitor fetal heart rate. The fetal heartbeat can be monitored using Doppler. Dopplers are useful for checking whether the fetus is growing normally, with a marked heartbeat in the fetus [2].

Heart rate in the fetus is very important because the fetal heart rate is a leading indicator of fetal life in the womb [1]. This is related to the use of Doppler which functions as a fetal heart rate monitor must be accurate. To test the accuracy of Doppler (Doppler calibrator) a fetal or fetal simulator is required.

The fetal simulator is an equipment used to perform tests and troubleshoot on Doppler [3]. Doppler as one of the medical equipment that is often used in health care institutions, in this case, it is necessary to pay attention to its performance such as functions and error factors so as not to experience a decline in function. According to the Regulation of the Minister of Health of the Republic of Indonesia Number: 363/Menkes/IV/1998 About Testing and Calibrating Medical Devices in Health Service Facilities Pasal 2 states that "Every medical equipment must be tested and/or calibrated to ensure the correct value of output or performance and safety of use" so that a fetal simulator or fetal Doppler simulator is an important equipment in determining the correctness of Doppler values.

This Fetal Doppler simulator was created by Ahmet MERT et al in 2013. It is equipped with 90, 102, 114, 126, 138, 150, 162, 174, 186 and 198 BPM selections. This equipment has been tested with Doppler on the market. and the maximum equipment error rate is $-1.4 \%$, so this equipment to be functional and has high accuracy to calibrate Doppler to prevent diagnostic errors in the world of health. To produce a fetal heartbeat sound, it use modified $24 \mathrm{~V}$ relay, but it must calculate the open and closed angles of the relay contacts [4], so that it is difficult to make mechanics on this equipment.

In 2014 Martha made a final assignment on "Fetal Simulator", this equipment use a selection of BPM with a range of 30-180 BPM with an increase of 30 BPM and mechanics used to produce fetal heartbeat sounds made of balloons. The equipment still has a disadvantage because it cannot be used to perform calibration when the fetal heart rate is above the range of 180 BPM. Another disadvantage of this equipment is that the sound of the resulting solenoid is less smooth.

The Fetal simulator made by Martha was developed by Nopa Ayu Solaikah in 2015 by making a "Fetal Doppler Simulator" equipment use a 60-210 BPM BPM selection with an increase of 30 BPM. Based on cases in the field, the fetus may experience supraventricular tachycardia (SVT) where the fetal heart rate ranges from 220-300 BPM [5], so a Doppler calibrator with a range above $210 \mathrm{BPM}$ is still recommended. In addition, fetal simulator circulating in health care institutions has a range of 30240 BPM with an increase of 30 BPM. Related to the description, the design made by Nopa Ayu Solaikah still need to be refined, so they can be used for the calibration process when the fetal heart rate is above 210 BPM.

The fetal heart rate in the Martha and Nopa design is produced by balloon mechanics. The balloon filled with water is then vibrated by a solenoid to produce a fetal heartbeat sound. However, balloons have a short lifetime because they are easy to lose volume and easily broken, so the balloon mechanics will make the fetal simulator performance less optimal as the mechanical performance decreases.

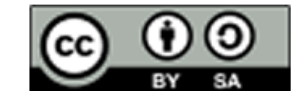

Attribution-ShareAlike 4.0 International 
Based on the identification of the above problems, the author intends to create an "Fetal Doppler Simulator Based Arduino" which is the development of previously made equipments by completing the BPM selection to 60-240 BPM with a $30 \mathrm{BPM}$ increase and changing the mechanical system that previously used balloons to make bottles the tool has a longer lifetime. In this study, the authors hope to make fetal Doppler simulators with the same value as fetal simulators that have been on the market.

\section{MATERIALS AND METHODS}

\section{A. Experimental Setup}

This study used Fetal Doppler (Philips / Avalon FM20 / SN: DE53017602) as DUT (Device Under Test). Data retrieval is done 6 times in each BPM point. The accuracy of the module is tested by comparing the results of the module with Fetal Simulator (Fluke Biomedical PS320).

\section{1) Materials and Tool}

This study used a solenoid that was modified with the top and bottom gave a tube and liquid to simulate the sound of the fetal heartbeat. The components used are Arduino Nano as a microcontroller, LCD $2 \times 16$ as a display.

\section{2) Experiment}

In this study, the author measured the output of the Arduino pin using an oscilloscope to know the frequency generated by the program at each BPM point and carried out measurements on the mechanics using Fetal Doppler to find out whether the BPM value produced was correct or not.

\section{B. The Diagram Block}

In this study, there are four setting buttons. After the Arduino setting is done, it will process data from the input settings to generate a signal with a certain frequency. The signal produced by Arduino enters the mechanical driver circuit to regulate the on / off of the mechanics so that the resulting mechanical beats are the same as the selected BPM value. The display shows the selected BPM value.

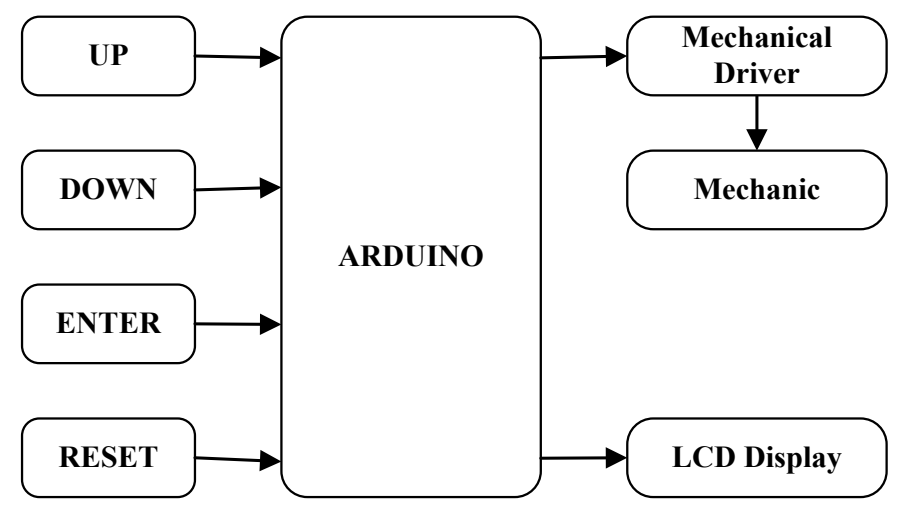

Fig. 1. The diagram block of the Fetal Doppler Simulator

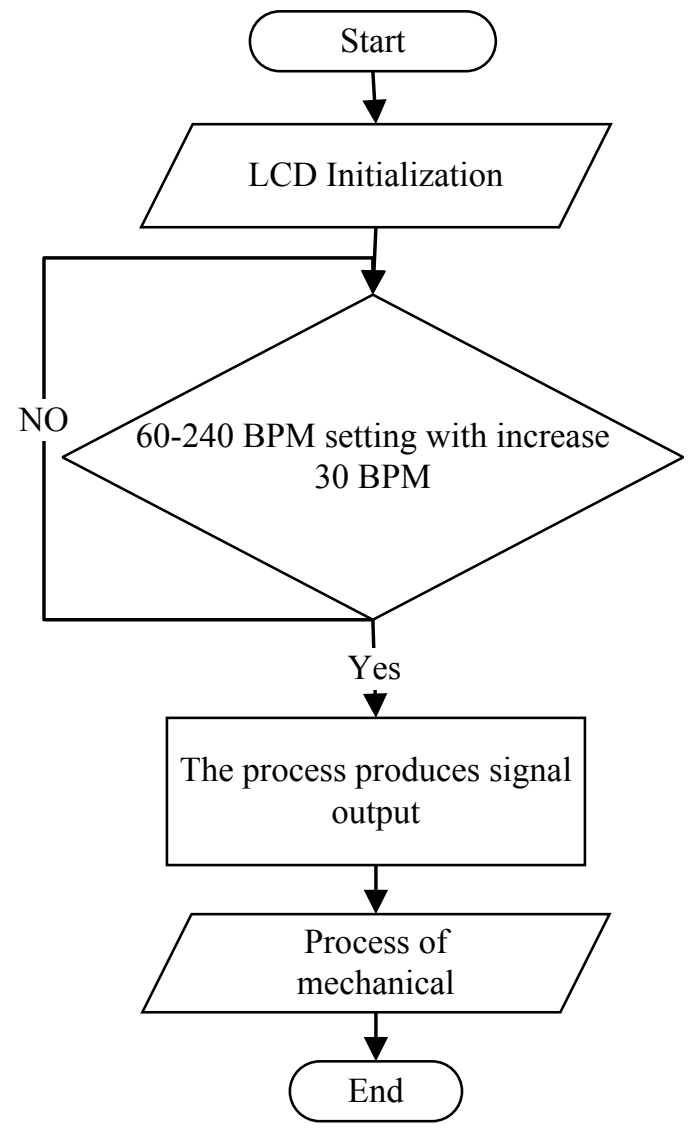

Fig. 2. The Flowchart of the Arduino Program

\section{The Flowchart}

The Arduino program was built on the flowchart as shown in Fig. 2. After LCD initialization, you can select the desired BPM value. After the BPM is selected, the Arduino will generate a signal with frequency according to the selected BPM value. The signal will move the solenoid to produce a beat with the value according to the selected BPM value.

\section{The Analog Circuit}

\section{1) Mechanical Driver}

A mechanical circuit driver as shown in Fig. 3 is a series that serves to regulate the solenoid on / off. This circuit consists of one transistor (BD139), one capacitor (1 microF) and one diode. The transistor functions as a switch so that the solenoid works according to the output signal from Arduino. Diode functions as a safety solenoid and transistor from backflow. Capacitors serve as short breaks so that the driver of the solenoid is expected to work optimally. 


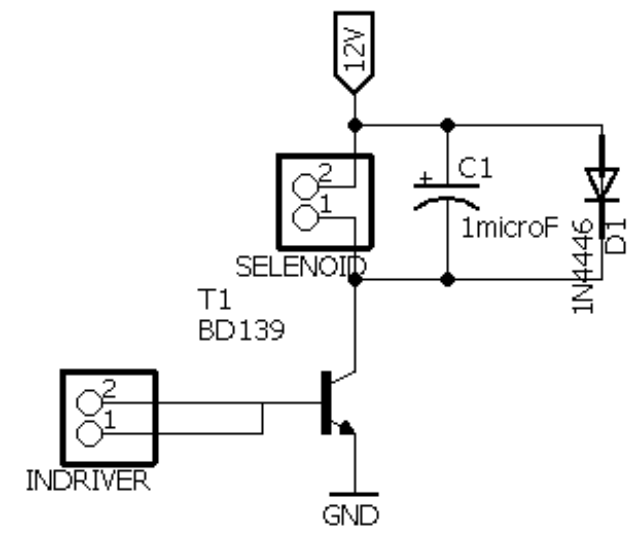

Fig. 3. Mechanical Driver

\section{RESULTS}

In this study, a trial was conducted on Fetal Doppler Simulator using Fetal Doppler to find out BPM produced by mechanical devices and a comparison of accuracy between the author's Fetal Doppler Simulator and Fetal Simulator (Fluke Biomedical Ps320) was carried out.
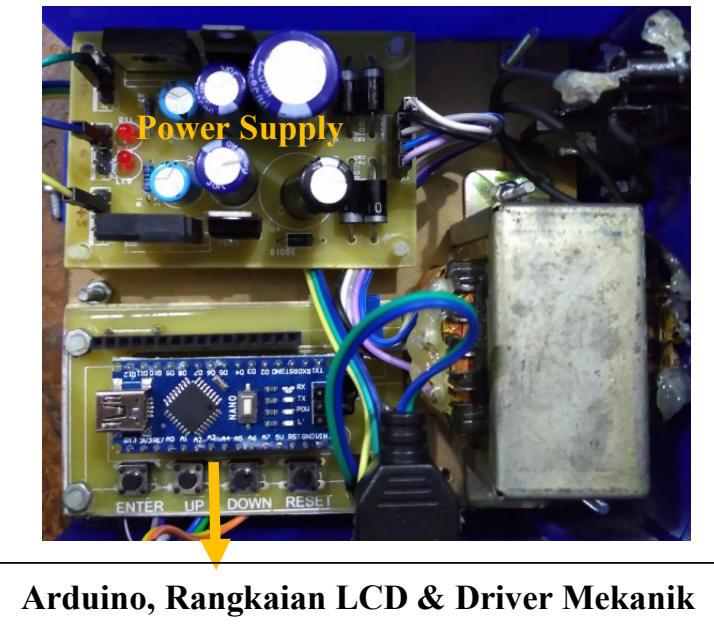

Fig. 4. The Control Part of Fetal Doppler Simulator

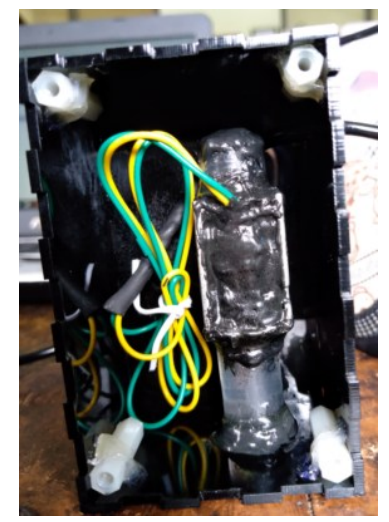

Fig. 5. Mekanik of Fetal Doppler Simulator
1) The Fetal Simulator Doppler Design

A picture of the control section and the mechanical part of the Fetal Doppler Simulator is shown in Fig. 4 and Fig. 5. The control section consists of a power supply circuit, Arduino nano which is the main of the Fetal Doppler Simulator, a series of LCDs and mechanical drivers. In the mechanical part, there is a solenoid that has been modified to simulate the sound of the fetal heartbeat.

\section{2) The Listing Program for the Selection of BPM}

In this study, Fetal Doppler Simulator is used BPM selection starting from 60-240 BPM with an increase of 30 BPM. Programs for BPM selection can be seen in Program Listing 1.

Listing program 1. Program for the Selection of BPM

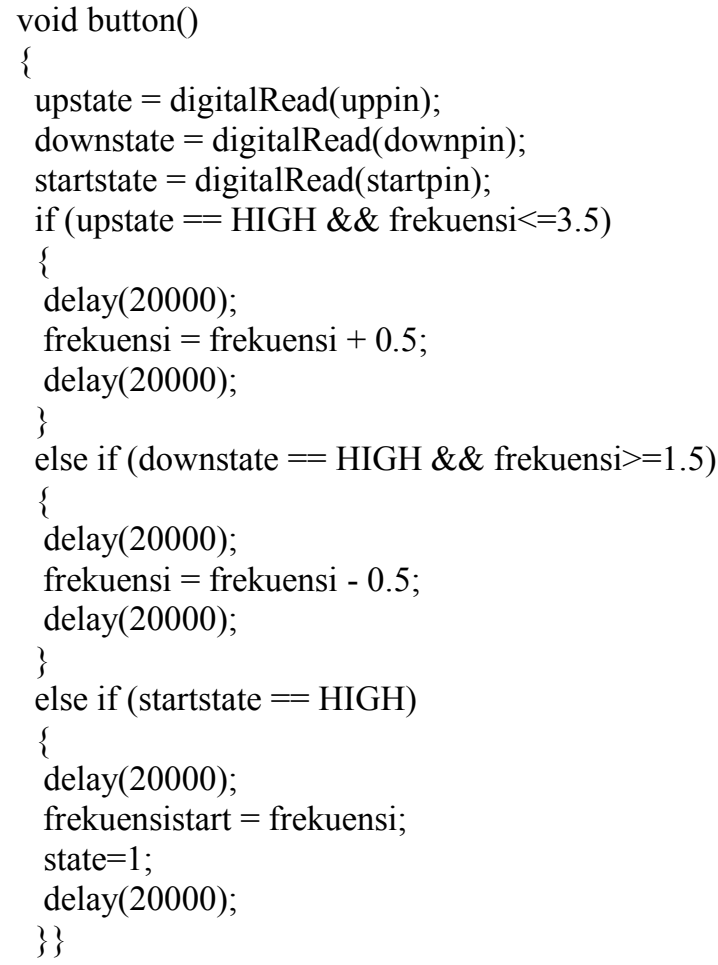

3) Delay Program to Produce BPM

Each BPM can be calculated the frequency value, from that frequency will get a period value. This period value is used to set Ton and Toff on the program so that BPM results as desired. Program listings from the program delay can be seen in Program Listing 2.

Listing Program 2. Delay Program to Produce BPM

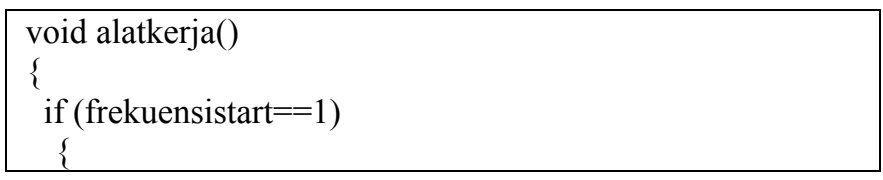




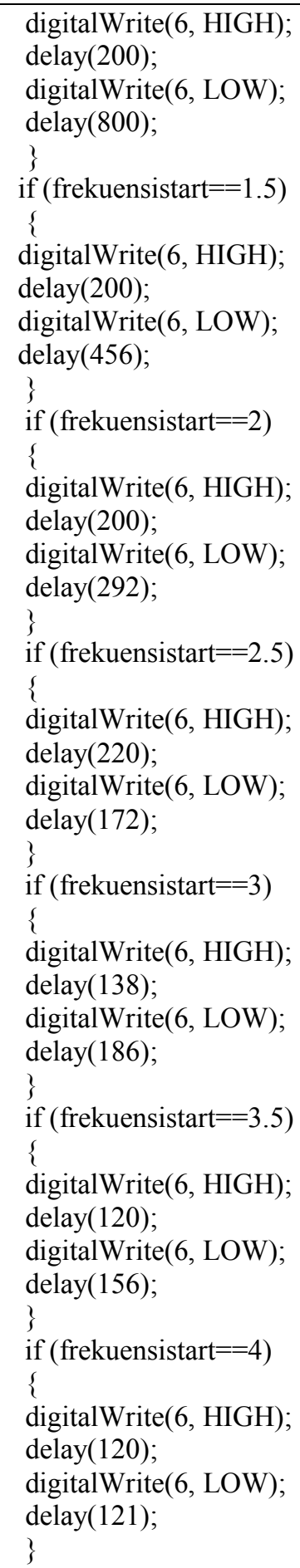

4) Fetal Doppler Simulator Measurement with Fetal Doppler

To determine the accuracy of the Fetal Doppler Simulator that has been made by the author, measurements were made using Fetal Doppler at each BPM point 6 times. The error value of the tool can be seen in Table I.
TABLE I. ERROR VALUE OF FETAL DOPPLER SIMULATOR.

\begin{tabular}{cc}
\hline BPM & Error(\%) \\
\hline 60 & 0 \\
\hline 90 & 0 \\
\hline 120 & 0 \\
\hline 150 & 0 \\
\hline 180 & 0 \\
\hline 210 & 0 \\
\hline 240 & 0.2 \\
\hline
\end{tabular}

5) Measurement of Fetal Simulator with Fetal Doppler

To find out the accuracy of the equipment that has been made, the authors compare the Fetal Doppler Simulator that has been made with Fetal Simulator (Fluke Biomedical Ps320). Both devices were measured using the same Fetal Doppler. Fetal simulators are also measured 6 times at each BPM point. Error-values from fetal simulator measurements can be seen in Table II.

TABLE II. ERROR VALUE OF FETAL SIMULATOR (FLUKE BIOMEDICAL PS320)

\begin{tabular}{cc}
\hline BPM & Error(\%) \\
\hline 60 & 0 \\
\hline 90 & 0 \\
\hline 120 & 0 \\
\hline 150 & 0 \\
\hline 180 & 0 \\
\hline 210 & 0.5 \\
\hline 240 & 0.2 \\
\hline
\end{tabular}

\section{DISCUSSION}

Based on measurements of Fetal Doppler Simulator, BPM results read by Fetal Doppler in the selection of BPM 60 to 210 have a $0 \%$ error. It means that between BPM settings on fetal Doppler simulators with BPM results that are read on fetal Doppler are the same. Whereas at BPM 240 there was an error of $0.2 \%$.

In fetal simulator measurements (fluke biomedical Ps 320) using the same fetal Doppler, the results of the error occurred at BPM 210 with a value of $0.5 \%$ while the 240 BPM was $0.2 \%$.

From Table I and Table II can be seen the difference in accuracy value between fetal Doppler simulator with fetal simulator (biomedical fluke Ps320) found at BPM points 210 and 240. In fetal Doppler simulators at BPM 210 errors are $0 \%$ while fetal simulators at BPM 210 error worth $0.5 \%$. While at 
point 240 the error value is both $0.2 \%$. This error value is still understandable because the tolerance of fetal Doppler calibration is $\pm 5 \%$. So it can be said that the fetal Doppler simulator made by the author has the same accuracy as the marketing tools (biomedical fluke PS320 brand).

\section{CONCLUSION}

This study shows the development of the Fetal Doppler Simulator from equipment that has been made before. This study was made with Arduino, a number of support sequences and solenoids that function to simulate fetal heartbeat sounds. This study produces Fetal Doppler Simulator with the same value of accuracy as fetal simulators on the market, especially biomedical fluke Ps320. In the future, this research can be developed by making it portable to make it more compact and easy to carry anywhere.

\section{REFERENCES}

[1] P. M. Hamilton, "Dasar-Dasar Keperawatan Maternitas, Edisi 6," in Dasar-Dasar Keperawatan Maternitas, EDISI 6, Jakarta, Buku Kedokteran ECG, 1995, p. 148.

[2] I. S. Faradisa, T. A. Sardjono and M. H. Purnomo, "Teknologi Pemantauan Kesejahteraan Janin," Seminar NasionalInovasi Dan AplikasiTeknologi Di Industri 2017, p. B32.5, 2017.

[3] M. B. "Fetal Simulator," in Fetal Simulator, 2006, Fluke corporation, 2007, pp. 1-13.

[4] A. MERT, M. SEZDI and A. AKAN, "A Test And Simulation Device For Doppler-Based Fetal Heart Rate Monitoring," Turkish Journal of Electrical Engineering \& Computer Sciences, pp. 1187-1194, 2013.

[5] A. Karataf, Z. Karatafl, T. Özlü, B. Küçükbayrak, S. E. Kilic and M. E. Dönmez, "Fetal Supraventricular Tachycardia," Perinatal Journal, pp. 5859, 2014. 